\title{
Upper Gastrointestinal obstruction as an Unusual Presentation of Thoracoabdominal Huge Aortic Aneurysm; a Case Report
}

\author{
Fatemeh Jahanshahi ${ }^{1}$ and Sam Zeraatian Davani ${ }^{1}$ \\ ${ }^{1}$ Iran University of Medical Sciences
}

June 6, 2021

\author{
Abstract \\ We reported a case with complaint of abdominal pain, nausea, vomiting, and oral intake intolerance due to a giant thoracoab- \\ dominal aortic aneurysm with contained leak and acute rupture within the circumferential mural thrombus which presented \\ with upper gastrointestinal obstruction manifestation. The rupture led to the patient undergoing emergency aneurysm repair. \\ Title: Upper Gastrointestinal obstruction as an Unusual Presentation of Thoracoabdominal \\ Huge Aortic Aneurysm; a Case Report \\ Fatemeh Jahanshahi $^{1^{*}}$ MD, Sam Zeraatian Nejad Davani ${ }^{2}$ MD \\ ${ }^{1}$ Student Research Committee, Faculty of Medicine, Iran University of Medical Science, Tehran, Iran \\ 2 Attending Professor of Cardiovascular Surgery and Dean of Faculty of Transplant and Organ Donation, \\ Iran University of Medical Sciences, Tehran, Iran \\ *Corresponding author: Fatemeh Jahanshahi \\ Rasool Akram Medical Complex, Niayesh Ave., Sattarkhan St., Tehran, Iran \\ Tel : +98 9124801231 \\ Fax: +982122826847 \\ Email : jahanshahi712@gmail.com
}

\section{Ethic approval and consent to participant:}

The present study is in compliance with ethical standards and standards of research involving humans. This article does not contain any studies involving animals performed by any of the authors.

\section{Consent for publication:}

Written informed consent was obtained from the patient for publication of this case report and any accompanying images. A copy of the written consent is available for review by the Editor-in-Chief of this journal.

\section{Availability of data and materials:}

Data in the current study are available from the corresponding author on reasonable request.

\section{Competing interests:}

There is no conflict of interest to declare. 


\section{Funding:}

This study has no financial source and support.

\section{Author's contributions:}

Study concept and design: SZ

Acquisition of data:FJ, SZ

Drafting of the manuscript: FJ, SZ

Critical revision of the manuscript for important intellectual content:SZ

Study supervision: FJ

All authors read and approved the final manuscript

\section{Acknowledgements:}

Title: Upper Gastrointestinal obstruction as an Unusual Presentation of Thoracoabdominal Huge Aortic Aneurysm; a Case Report

\section{Abstract:}

We reported a case with complaint of abdominal pain, nausea, vomiting, and oral intake intolerance due to a giant thoracoabdominal aortic aneurysm with contained leak and acute rupture within the circumferential mural thrombus which presented with upper gastrointestinal obstruction manifestation. The rupture led to the patient undergoing emergency aneurysm repair.

Key words: aortic aneurysm; aneurysm; thoracoabdominal aortic aneurysm; huge; rupture; gastric obstruction

\section{Key clinical message:}

Though upper gastrointestinal obstruction is an extremely rare manifestation of giant thoracoabdominal aneurysm, it should be considered as a differential diagnosis in similar cases.

\section{Introduction:}

An aortic aneurysm with the diameter of more than $10 \mathrm{~cm}$ is considered giant. TAAAs without treatment can be life-threatening. In these cases, the rupture has high mortality and its risk is approximately $60 \%$ $[1,2]$. $43 \%$ of all TAAAs are asymptomatic and discovered accidentally during radiologic investigations for an irrelevant cause. Among other $57 \%$ of cases, which are symptomatic, $9 \%$ become symptomatic when the aneurysm ruptures. Pulsetile mass in the abdominal wall is the specific symptom of aortic aneurysm; however, other symptoms are non-specific and caused by extrinsic compression of the giant aneurysm into the surrounding structures. Giant aneurysms can compress the gastrointestinal tract and cause an obstruction with symptoms including vomiting, constipation, abdominal distention, and pain.[1,3] This article reports a case with symptoms of abdominal pain, nausea, vomiting, and oral intake intolerance in the past 4 days before admission, and moreover with protruded abdomen with pulsation. In radiologic investigations, a giant thoracoabdominal aortic aneurysm (TAAA) with chronic contained leak and acute rupture within the circumferential mural thrombus were observed. The patient was resuscitated, transferred to emergency operation room, and underwent aorto-renal reconstruction surgery.

\section{Case Presentation:}

The patient was a seventy-year-old man who went to the emergency department with complaint of acute abdominal pain, nausea, vomiting, and oral intake intolerance during the last 4 days. 
The patient had abdominal pain with preference of epigastric region radiated to back in the past 4 days before admission which became intensively severe over the last few hours. In both inspection and palpation, significant findings which stood out were a protuberant abdomen with expansile pulsation.

He stated that over the last year he felt a periumbilical mass and pulsation, however, he did not go to a doctor and follow up.

In his medical history, he had been smoking for 50 years, 1 pack daily, and no diabetic mellitus, hypertension, ischemic heart disease, and dyslipidemia were reported. At the time of admission, vital signs including respiratory rate $=17$ per minute, pulse rate $=112$ per minute, blood pressure right hand $98 / 48 \mathrm{~mm} \mathrm{Hg}$ and left hand $99 / 46 \mathrm{~mm} \mathrm{Hg}, \mathrm{O} 2$ saturation $=95 \%$, and temperature $=36.6^{\circ} \mathrm{C}$. In superficial palpation, it was observed that the abdomenprotruded and the patient had tenderness in the epigastric region and did not allow deep touching. No bowel sound was osculated in 4 quadrants of the abdomen in one minute.

Laboratory tests of the patient from initial blood sample showed: $W B C=14.4(103 / \mu \mathrm{L})$ (normal range $4-10), \mathrm{Hb}=11.3(\mathrm{~g} / \mathrm{dL})$ (normal range 14-18), Platelet $=136\left(10^{3} / \mathrm{mm}^{3}\right)$ (normal range 140-440), PT = 18.2 seconds, $\mathrm{PTT}=36$ seconds, and $\mathrm{INR}=1.46, \mathrm{BUN}=29.1 \mathrm{mg} / \mathrm{dl}, \mathrm{Cr}=2.20, \mathrm{Na}=149, \mathrm{~K}=4.1 \mathrm{mg} / \mathrm{dl}$, $\mathrm{AST}=117, \mathrm{ALT}=110$, Bilirubin total $=1.8$ and direct $=0.5$

In the emergency abdominopelvic sonography, which conducted for the patient in the emergency room, evidence of a thoracoabdominal aortic aneurysm was observed. The patient with systolic blood pressure of $70 \mathrm{~mm} \mathrm{Hg}$ underwent spiral thoracoabdominal CT scan with IV contrast. In tomography report, a giant aneurysmal dilation of the aorta from diaphragm aortic hiatus continued downward to infrarenal aorta with diameter of $103 \times 148 \mathrm{~mm}$ and approximate length of $164 \mathrm{~mm}$ containing a huge mural thrombus. Additionally, evidence of contrast leak from aneurismal sac was noted. Moreover, a huge hematoma in the right retroperitoneal space pushing the right kidney forward and compressing the bowel and the collapse of the stomach were reported. (Figure1)

Laboratory tests of the patient from second blood sample showed $\mathrm{Hb}=2.6$ which demonstrated an acute bleeding due to the rupture of aneurysm sac. The patient was resuscitated with 4 units of packed red blood cells and fresh frozen plasma and transferred to the emergency operation room. He underwent induction for general anesthesia with ketamine and maintenance with fentanyl, atracurium, and midazolam. The patient blood pressure was under control between $70-80 \mathrm{~mm} \mathrm{Hg}$ with nepride and vasopressin.

Midline incision was performed and proximal aortic control achieved at diaphragmatic hiatus. The Aneurysm sac was incised, and the huge hematoma was removed and sent to the pathology.(Figure2) And aorto-renal reconstruction surgery was performed on the patient. (Figure3) After uneventful recovery, he was transferred to intensive unit care with norepinephrine drip. Finally, the patient completed an uneventful post-operation course.

\section{Discussion:}

Giant aneurysm of the aorta is defined as an aneurysm with a diameter of more than $10 \mathrm{~cm}$. In general population, occurrence of giant aneurysm is not prevalent. On the other hand, presence of one of the giant aneurysm etiologies including connective tissue disorder such as Marfan and Ehlers-Danlos syndromes, infection with syphilis, tuberculosis and HIV, atherosclerotic vascular diseases, and giant cell arthritis make the formation and progress of giant aneurysms more probable. Aneurysms can be completely asymptomatic and discovered accidentally. However, giant aneurysms can cause nonspecific symptoms due to their compression effect in to the surrounding organs. Moreover, the symptoms of some aortic aneurysm cases appear when they rupture or are dissected suddenly. The risk of rupture of the aneurysm is directly affected by the size of the aneurysm. The probability of rupture in aneurysms with diameters of equal or greater than $8 \mathrm{~cm}$ is almost 40\%.[1, 4]Rupture of aneurysm has a spectrum ranging from contained rupture to flank rupture of aneurysm which can empty the patient's cardiovascular capacity in few minutes. TAAA can be repaired by endovascular or open aortic construction approaches. However, in ruptured cases, open surgery is a definite option for the survival of the patient. 
For a thorough examination and making a correct diagnosis, computed tomography scan with contrast and CTA have necessary specificity and sensitivity for detecting detailed information about mural thrombus, hematoma, aneurysm wall, leakage, or rupture of aneurysm. [3, 5]

In the present case, we encountered a giant chronic TAAA with contained leak which led to formation of circumferential mural thrombus in addition to an acute frank rupture of aneurysm within the thrombus. This case presented with upper gastrointestinal obstruction manifestation and was managed with resuscitation and an emergency aorto-renal reconstructive surgery.

\section{List of abbreviations:}

CT: computed tomography

IV: intravenous

TAAA: thoracoabdominal aortic aneurysm

\section{References:}

[1] Maleki, Farid, Fatemeh Jahanshahi, and Jalal Vahedian-Ardakani. "Giant saccular abdominal aortic aneurysm with manifestation of gastric inlet obstruction mimicking a fundal gastrointestinal stromal tumor: A report on an unexpected case." Clinical Case Reports. [2] Di Marco, Luca, Domenico Attina, and Davide Pacini. "Giant thoracoabdominal aortic aneurysm." Journal of Cardiovascular Medicine 19.1 (2018): 34-35. [3] Deng, Jianying, and Wei Liu. "A 52-year-old man with asymptomatic giant thoracic-abdominal aortic aneurysm." Journal of Cardiac Surgery (2021). [4] Frederick, John R., and Y. Joseph Woo. "Thoracoabdominal aortic aneurysm." Annals of cardiothoracic surgery 1.3 (2012): 277. [5] Kusadokoro, Sho, et al. "Dissected thoracoabdominal aortic aneurysm repair with modified parallel endografting." Journal of Cardiac Surgery 35.11 (2020): 3220-3223.

\section{Figure legends:}

Figure 1. Aand B: Thoracoabdominopelvic CT scan with IV contrast, axial view: a giant aneurysmal dilation of the aorta with diameter of $103 \times 148 \mathrm{~mm}$ and approximate length of $164 \mathrm{~mm}$ containing a huge mural thrombus.(white arrow) Additionally, Contrast leak from aneurismal sac showed with blue arrow, and a giant hematoma in the right retroperitoneal space (red arrow) pushing the right kidney forward and collapse the stomach.(white star)

Figure 2: Gross specimen of giant organized hematoma.

Figure 3: Perioperative figure of aorto-renal reconstruction.

\section{Hosted file}

figure1.docx available at https://authorea.com/users/417979/articles/525040-uppergastrointestinal-obstruction-as-an-unusual-presentation-of-thoracoabdominal-huge-aorticaneurysm-a-case-report

\section{Hosted file}

figure 2.docx available at https://authorea.com/users/417979/articles/525040-uppergastrointestinal-obstruction-as-an-unusual-presentation-of-thoracoabdominal-huge-aorticaneurysm-a-case-report

\section{Hosted file}

figure 3.docx available at https://authorea.com/users/417979/articles/525040-uppergastrointestinal-obstruction-as-an-unusual-presentation-of-thoracoabdominal-huge-aorticaneurysm-a-case-report 